\title{
Molecular analysis of single isolated glands in gastric cancers and their surrounding gastric intestinal metaplastic mucosa
}

\author{
TAMOTSU SUGAI $^{1}$, WATARU HABANO ${ }^{2}$, YU-FEI JIAO ${ }^{1}$, MINORU TOYOTA $^{3}$, HIROMU SUZUKI ${ }^{3}$, \\ MITSUNORI TSUKAHARA ${ }^{1,4}$, HITOHIKO KOIZUKA ${ }^{1,4}$, RISABURO AKASAKA ${ }^{1,4}$, \\ KEISUKE KOEDA ${ }^{5}$, GO WAKABAYASHI ${ }^{5}$ and KAZUYUKI SUZUKI ${ }^{4}$ \\ ${ }^{1}$ Department of Pathology, Division of Diagnostic Molecular Pathology, School of Medicine, \\ ${ }^{2}$ Department of Pharmacodynamics and Molecular Genetics, School of Pharmacy, Iwate Medical \\ University, Iwate; ${ }^{3}$ Department of Biochemistry, School of Medicine, Sapporo Medical University, \\ Sapporo; ${ }^{4}$ Department of Internal Medicine, Division of Gastroenterology and Hepatology, \\ ${ }^{5}$ Department of Surgery, School of Medicine, Iwate Medical University, Iwate, Japan
}

Received June 9, 2009; Accepted August 7, 2009

DOI: 10.3892/or_00000602

\begin{abstract}
The biological properties and underlying genetics of gastric cancer and gastric intestinal metaplasia evolve with neoplastic progression from the genetics of the original gland cell. PCR assay with crypt isolation was used in tumors from 20 patients to examine microsatellite alterations (allelic imbalance at 17p, 5q, 18q, 3p, 4p, and 9p, and microsatellite instability) in glands from each tumor and from intestinal metaplastic lesions. Tumor specimens were processed as either pooled-gland samples or single-gland samples. Pooled gland sample was composed of 10-20 tumor glands, intestinal metaplastic glands, or nonmetaplastic glands. Single gland sample was 10 tumor glands from tumor and single gland sample was 5 gastric intestinal metaplastic and 5 nonmetaplastic glands from its surrounding metaplastic mucosa. Multiple genetic alterations were found in individual tumor glands, with various subclonal expansions seen within the same tumor. Although microsatellite instability was found in 2 of 20 tumor single-gland samples, none was detected in metaplastic single-gland samples. Most cancers appear to have a heterogeneous composition. On the other hand, microsatellite alterations were also detected within the nonmetaplastic as well as intestinal metaplastic single-gland samples. In conclusion, the present data on tumor and corresponding intestinal metaplastic and nonmetaplastic glands suggest that genetic alterations already occur within the surrounding of the noncancerous mucosa.
\end{abstract}

Correspondence to: Dr Tamotsu Sugai, Division of Diagnostic Molecular Pathology, Department of Pathology, 19-1 Morioka, Iwate 020-8505, Japan

E-mail: tsugai@cocoa.ocn.ne.jp

Key words: crypt isolation, gastric cancer, intestinal metaplasia, loss of heterozygosity, microsatellite instability

\section{Introduction}

It has long been established that gastric cancers develop from a single gland cell that undergoes an accumulation of genetic changes (1), and which eventually results in a malignant tumor with monoclonal character $(2,3)$. Recent molecular genetic evidence also supports this concept (1). Despite the monoclonal origin of such cancerous tumors, any single tumor consists of numerous tumor glands, which may have various genetic alterations relative to the cell of origin. According to established theory, these accumulated genetic alterations can be identified by loss of heterozygosity $(\mathrm{LOH})$ at many chromosomal loci within the tumor (4). It is also true that differences between tumor glands are thought to cause genetic heterogeneity within the same tumor (1-3), and some studies stated that genetic heterogeneity is frequently found in individual gastric cancers (5-7). Such genetic heterogeneity within the same tumor complicates the development of a tumor-treatment strategy based on tumor pathogenesis $(8,9)$. Analyzing the accumulation of multiple genetic alterations in a single tumor gland is thought to be useful for assessing genetic differences among the cells of a single tumor $(2,3)$.

Gastric intestinal metaplasia (GIM) is frequently found in the mucosa surrounding a gastric cancer (10), and is commonly thought to be a precancerous condition for gastric cancer, especially differentiated-type gastric cancers $(11,12)$. However, an alternative hypothesis, the 'paracancerous' condition, as distinct from the 'precancerous' condition, has been described, especially in Japan (13). The paracancerous hypothesis derives from the fact that, based on routine pathological examination of surgical specimens, no pathologist has yet been able to identify the feature proving that differentiated-type gastric carcinomas arise directly from intestinal metaplastic glands. Accordingly, it remains unclear whether GIM is truly a precancerous lesion. In addition, GIM is not a single entity but rather a heterogeneous group of metaplastic glands (14). Previous studies suggested that GIM by itself is associated with carcinogenesis of differentiated-type gastric cancer and that GIM has a heterogeneous composition with different 
biological characteristics (14-17). Identification of 1 or more biomarkers to reliably differentiate GIM types associated with gastric cancer and the molecular alterations associated with various types of GIM may be very valuable clinically as a tool for identifying patients who may be at higher risk for gastric cancer. However, molecular alterations contributing to the development or neoplastic progression of GIM have not been clarified (18). In addition, a non-neoplastic gland which is seen in the gastric intestinal metaplastic mucosa is not necessarily an intestinal metaplastic gland. Nonmetaplastic glands are often observed in gastric metaplastic mucosa. It is likely that the nonmetaplastic glands are also associated with gastric carcinogenesis. However, the molecular alterations of nonmetaplastic glands within the gastric intestinal metaplastic mucosa are still not identified.

For genetic evaluations of an individual gland taken from tissue of a single gastric cancer and the surrounding GIM and nonmetaplastic glands (intestinal metaplastic and non-intestinal glands), it is necessary to isolate single glands from the larger tissue mass. The crypt isolation method can be used to obtain individual tumor and non-neoplastic glands from a given tumor or surrounding tissue $(19,20)$. In the current study, we used crypt isolation methodology to examine molecular alterations of single tumor glands from a gastric cancer and single glands from surrounding GIM tissue. The aim of this study was to verify the role of intratumoral molecular differences in sporadic gastric cancer and to further our understanding of molecular alterations in GIM tissue that result in gastric tumorigenesis.

\section{Materials and methods}

Tissue samples. Tissues were obtained from 20 patients with sporadic primary gastric adenocarcinoma of differentiated type, who had undergone gastrectomy. Of the 20 patients, there were 17 men and 3 women (mean age: 64.2 years). Tumor histological type and stage were classified according to the Japanese Research Society criteria for cancer of the stomach (21). The location of the gastric cancer was determined and the tumors were subclassified into 2 groups: proximal or distal. Clinicopathological data for the 20 patients in our study are shown in Table I. In addition, in order to clarify genetic alterations in non-neoplastic glands not demonstrating intestinal metaplasia (nonmetaplastic gland), nonmetaplastic glands associated with gastric adenocarcinomas were analyzed in the 10 tumors in which they were available.

Fresh tumor specimens and adjacent tissue were obtained from resected gastric cancers. Gastric mucosa most distal to the tumor that was regarded as normal was removed from the submucosa with scissors and discarded. In contrast, intestinal metaplastic mucosa was obtained from antral mucosa. Tissue tumor samples were obtained primarily from the central area of the tumor.

Crypt isolation technique. Isolation of tumor and mucosal glands was performed as previously described (22). Briefly, fresh tumor and non-neoplastic mucosa were separated from the underlying tissue layer and cut with a razor into minute pieces, and then incubated at $37^{\circ} \mathrm{C}$ for $30 \mathrm{~min}$ in Hanks' balanced salt solution (HBSS), which is calcium- and
Table I. Clinicopathological findings of gastric cancers examined.

Total 20

Gender (male/female)

Age (mean)

Locus

Proximal

2

Distal

18

Histological type

WDA

8

MDA

Stage

I 3

II 4

III 10

IV 3

WDA, well differentiated adenocarcinoma; MDA, moderately differentiated adenocarcinoma.

magnesium-free, containing $30 \mathrm{mmol} / \mathrm{l}$ ethylenediaminetetraacetic acid (EDTA). Specimens were stirred in HBSS for 30-40 min to allow isolation of cancerous and normal glands from the lamina propria mucosa or fibrous stroma. Isolated specimens were immediately fixed in $70 \%$ ethanol and stored at $4^{\circ} \mathrm{C}$ until analysis.

Representative cancerous glands are shown in Fig. 1a-f. To obtain intestinal metaplastic glands, nonmetaplastic glands were isolated from antral mucosa [stained with Alcian blue ( $\mathrm{pH} 2.5)$.

Identification of isolated gastric intestinal metaplastic glands. In the present study, GIM was recognized by the presence of goblet cells stained by Alcian blue. Detailed histological identification of intestinal metaplasia was performed on sections of paraffin-embedded tissue located adjacent to the tissue used for crypt isolation. Most tissue sections showed incomplete intestinal metaplasia.

Identification of isolated gastric intestinal nonmetaplastic glands. Nonmetaplastic glands which were not stained by Alcian blue were isolated from antral mucosa. These glands could be easily distinguished from metaplastic glands. However, nonmetaplastic glands were obtainable in only 10 of 20 cases.

Identification of isolated normal gastric glands. Normal mucosal tissue was defined as glands obtained from Alcian blue-negative fundic mucosa, and was confirmed by histological examination. Normal mucosa was used to examine microsatellite alterations in normal (negative) controls.

DNA extraction. Isolated tumor glands were handled as follows: Ten to 20 isolated glands (tumor, intestinal metaplastic, and nonmetaplastic glands) were obtained from each 

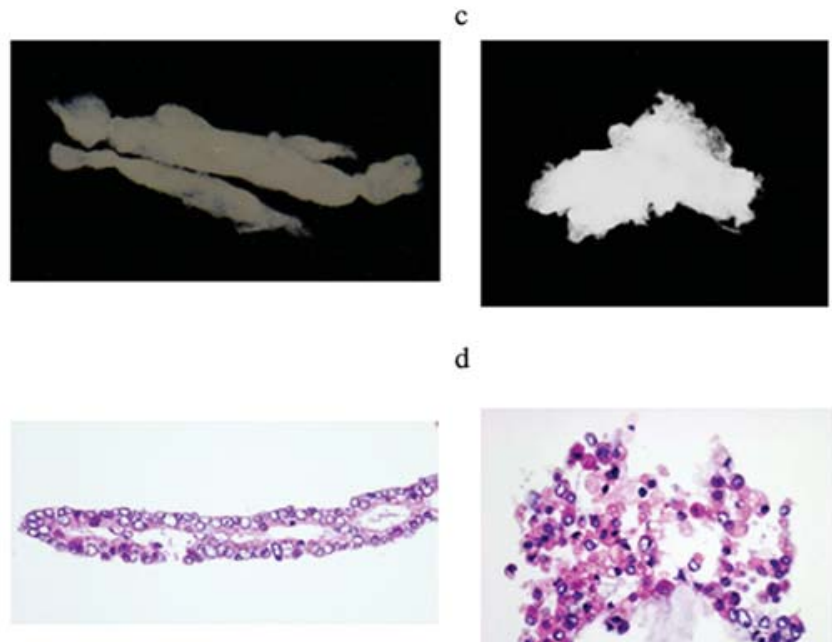

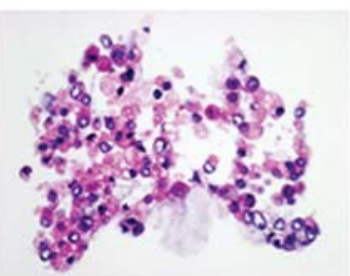

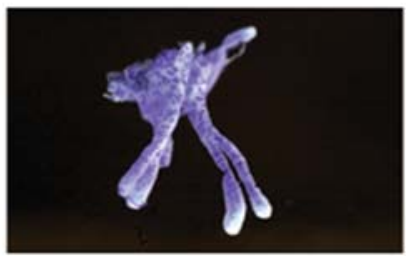

g

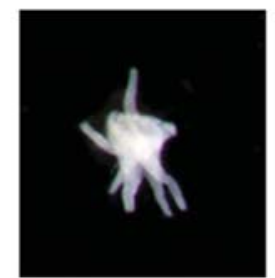

f

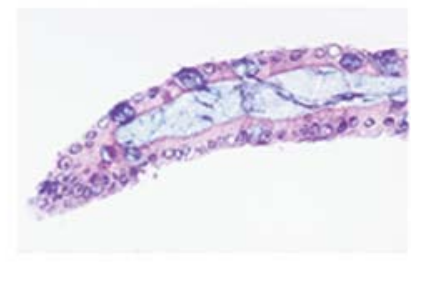

Figure 1. Well-differentiated adenocarcinoma. (a) As seen under a dissecting microscope and (b) with hematoxylin and eosin staining (H\&E). A single moderately differentiated tumor gland (c) under a dissecting microscope and (d) as seen with H\&E staining. Single intestinal metaplastic gland (e) as stained by Alcian blue ( $\mathrm{pH}$ 2.5) under a dissecting microscope and visualized with H\&E staining (f). Single non-intestinal metaplastic gland (g).

Table II. Frequencies of allelic imbalances at each locus in tumor pooled-gland samples.

\begin{tabular}{lcc}
\hline & Informative cases & Pooled-gland sample (\%) \\
\hline $17 \mathrm{p}$ & 15 & $8(53.3)$ \\
$5 \mathrm{q}$ & 18 & $10(55.6)$ \\
$18 \mathrm{q}$ & 18 & $8(44.4)$ \\
$3 \mathrm{p}$ & 18 & $8(44.4)$ \\
$4 \mathrm{p}$ & 18 & $10(55.6)$ \\
$9 \mathrm{p}$ & 14 & $13(92.6)$ \\
\hline
\end{tabular}

tissue sample. DNA extraction was performed on these glands to create the pooled-gland samples (3). Separately, 10 tumor glands and 5 intestinal metaplastic glands or nonmetaplastic glands were obtained from each tumor examined and its surrounding antral mucosa. DNA was extracted from each individual tumor, intestinal metaplastic, and nonmetaplastic gland, respectively, to create the single-gland samples, using techniques described previously $(2,3)$. The DNA concentration of single tumor glands was estimated by the TagMan realtime PCR method using the ribosomal protein P0 (3684) gene as a reference (3). In addition, the amount of DNA content that we examined varied from 98.6 to $1034.8 \mathrm{ng}$ (mean: $410.1 \mathrm{ng}$ ). On the other hand, that of DNA content examined in single intestinal metaplastic glands varied from 112.6 to $253.4 \mathrm{ng}$ (mean: $198.7 \mathrm{ng}$ ).

Microsatellite analysis. Microsatellite analysis included 15 microsatellite markers (2 mono- and 13 dinucleotide repeats). These markers were selected either because of their location at chromosomal sites in or near genes known to be involved in gastric carcinogenesis ( $3 p, 4 p, 5 q, 9 p, 17 p$, and 18q), or because they are very sensitive markers for determination of microsatellite instability (MSI) (BAT25 and 26). The markers used for analysis of LOH in this study were: 3p (D3S2402,
D3S1234), 4p (D4S2639, D4S1601), 5q (D5S107, D5S346, D5S299, D5S82), 9q (D9S171, D9S1118), 17p (TP53), and 18q (D18S487, D18S34).

PCR reactions were performed as described previously $(23,24)$. One of the primers used for amplification was fluorescently labeled. PCR products were separated and detected with an automated sequencing system as previously reported $(19,23)$.

Scoring of allelic imbalance. Allelic imbalance (AI) was determined using a calculation method described previously (25). A tumor was considered to have AI if the allelic peak ratio was $<0.6$, representing an allelic signal reduction of at least $40 \%$. We interpreted this allelic imbalance as LOH with the provision that, in some cases, the change in allele peak ratio may have resulted from allelic amplification. When AI was observed in at least 1 locus of the markers examined, imbalances of the examined loci were confirmed. Finally, tumors exhibiting MSI at a given locus were not evaluated for $\mathrm{LOH}$.

The overall extent of $\mathrm{LOH}$ for each single-gland sample (tumor, intestinal metaplastic, or nonmetaplastic single-gland sample) was calculated as follows: the number of single glands showing $\mathrm{LOH}$ divided by the number of informative (excluding uninformative cases, samples showing MSI, and cases that were not examined) single glands for each tumor or each intestinal metaplastic gland sample.

Scoring of microsatellite instability. An additional peak in tumor DNA compared with nontumor tissue was classified as instability for the marker examined. Instability in BAT 25 or 26 was defined as MSI positive (26).

\section{Results}

In the present study, PCR analysis was performed reproducibly and there were no PCR failures, as has been described elsewhere (3). The frequencies of allelic imbalance at the 

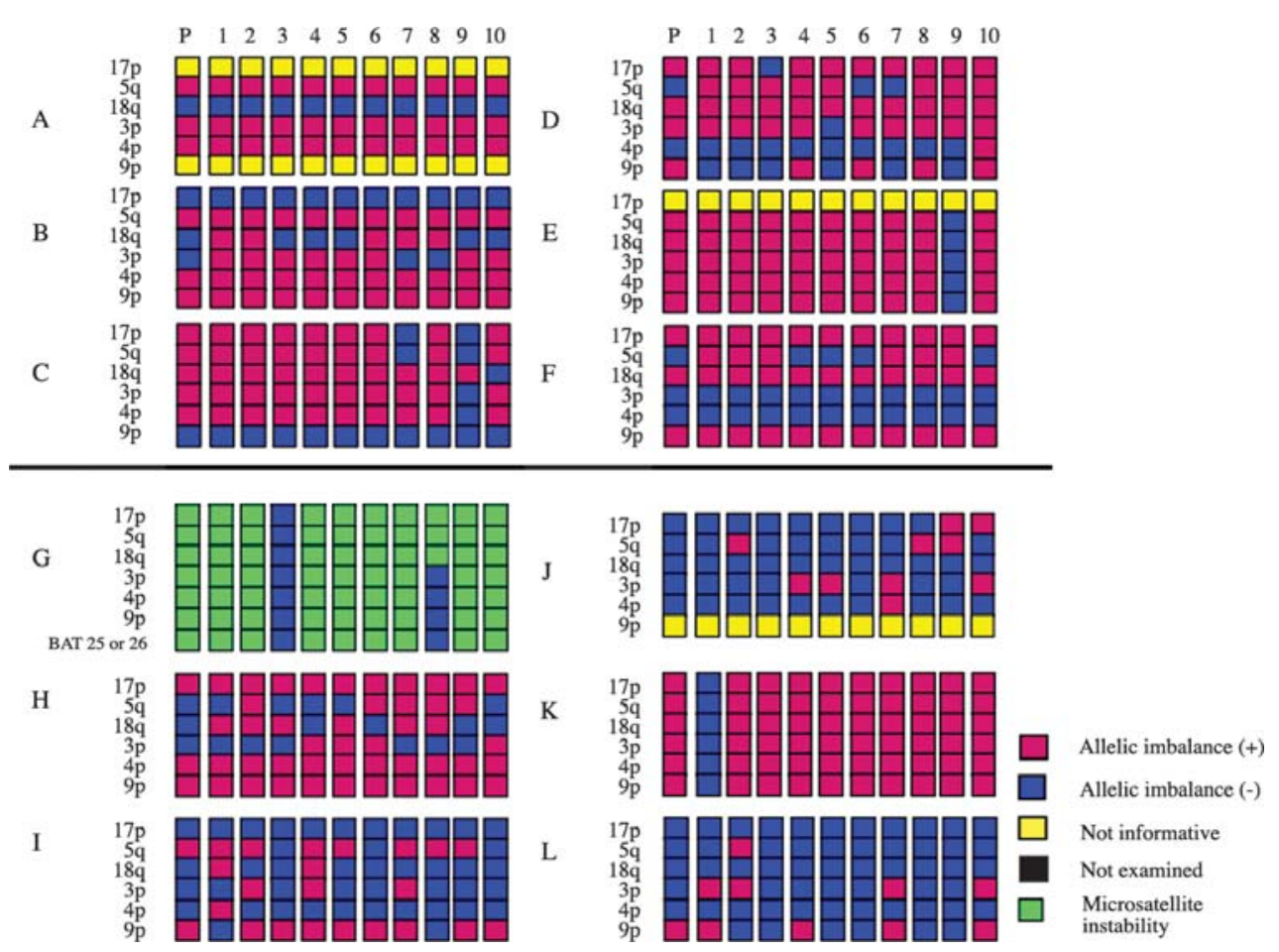

Figure 2. A detailed view of allelic imbalances on 5q, 17p, 18q, 3p, 4p, 9p, and microsatellite instability in pooled-tumor samples and in corresponding tumor single-gland samples (cases: A-L). P, pooled gland sample; LOH, loss of heterozygosity; N, negative; NI, not informative; NA, not amplified; MSI, microsatellite instability.

$\begin{array}{llllllllllllll}P & 1 & 2 & 3 & 4 & 5 & 6 & 7 & 8 & 9 & 10\end{array}$
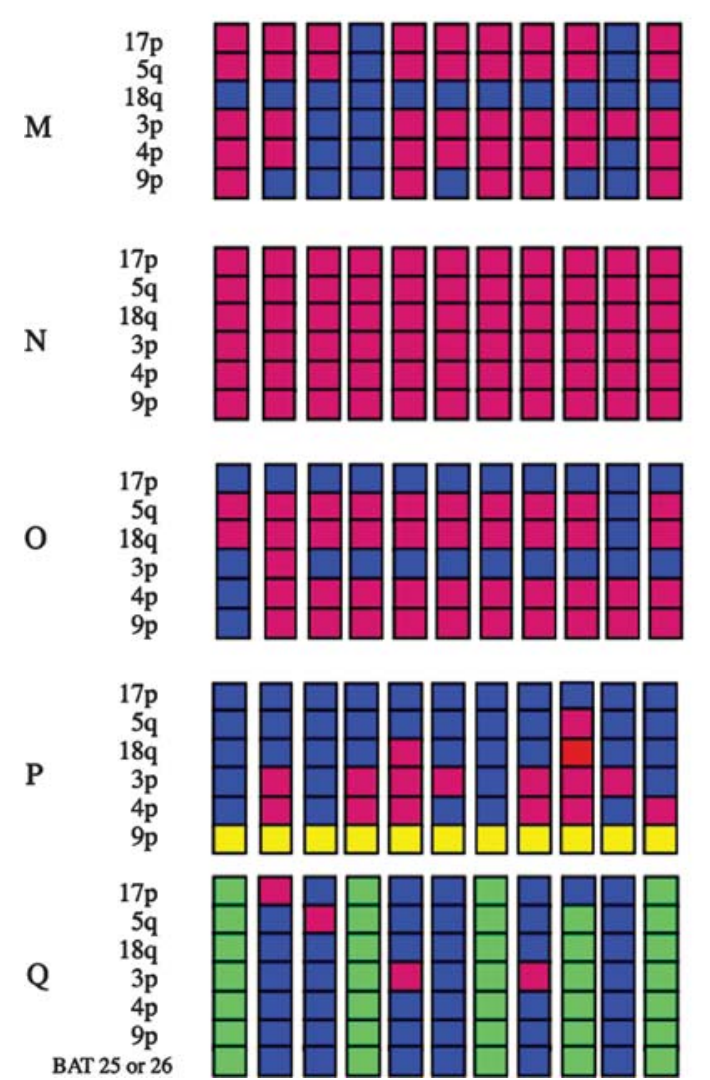

$\begin{array}{llllllllllllll}P & 1 & 2 & 3 & 4 & 5 & 6 & 7 & 8 & 9 & 10\end{array}$
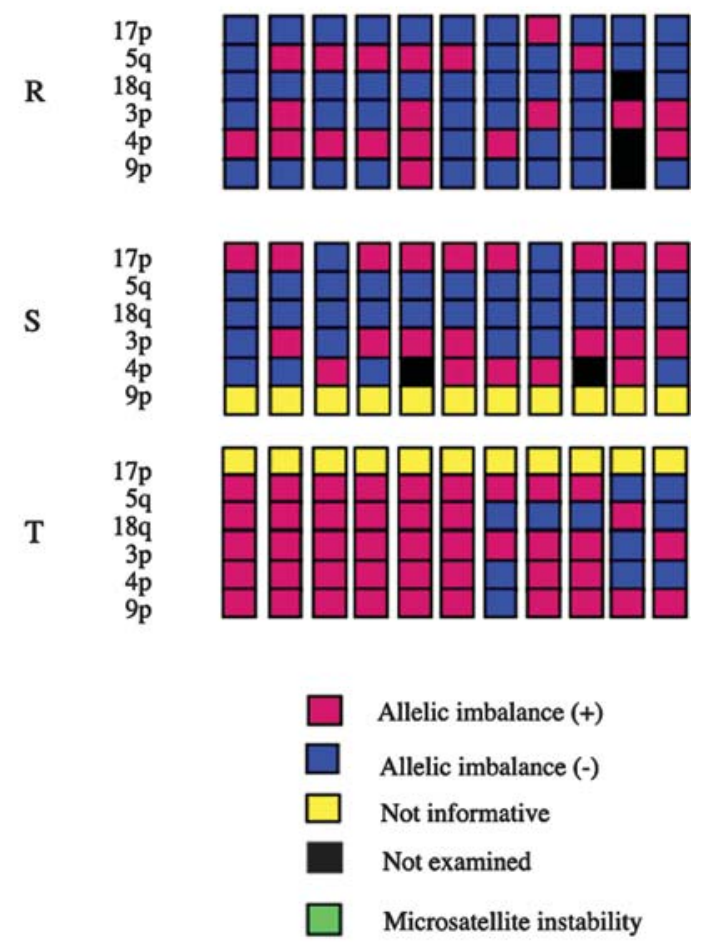

Figure 3. A detailed view of allelic imbalances on 5q, 17p, 18q, 3p, 4p, 9p, and microsatellite instability (MSI) in pooled-tumor samples and in corresponding tumor single-gland samples (cases: M-T). P, pooled gland sample; LOH, loss of heterozygosity; N, negative; NI, not informative; NA, not amplified; MSI, microsatellite instability. 

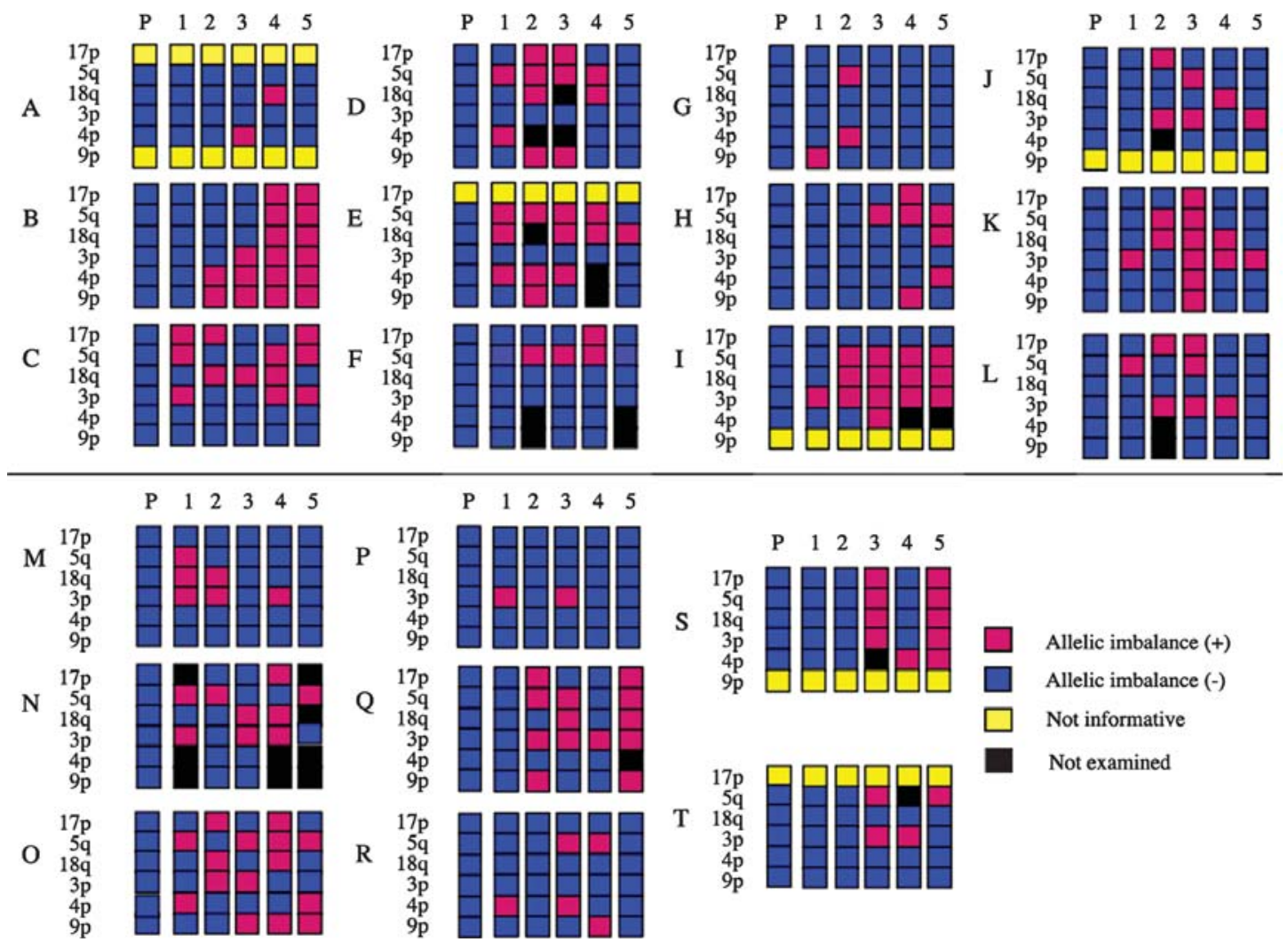

Figure 4. A detailed view of allelic imbalances on 5q, 17p, 18q, 3p, 4p, 9p, and microsatellite instability in intestinal metaplastic pooled-gland samples and in corresponding intestinal metaplastic single-gland samples (samples: A-T). Multiple genetic alterations were seen in the single intestinal metaplastic gland samples. P, pooled gland sample; LOH, loss of heterozygosity; N, negative; NI, not informative; NA, not amplified.

chromosomal loci that were studied in the tumor pooledgland samples are listed in Table II. We defined genotype as the AI pattern of each examined sample.

Concordance of genotype of pooled-gland samples and singlegland samples. We examined the genotype concordance of pooled-gland samples and the predominant genotype in corresponding single-gland samples. In 4 cases there were different AI patterns seen in the tumor pooled-gland samples and in those of the corresponding tumor single-gland samples (samples B, H, O, and S; Figs. 2 and 3). In addition, in another 7 cases (samples D, F, I, J, L, P, and R; Figs. 2 and 3), the predominant genotypes in tumor single-gland samples were different from those of the corresponding pooled-gland samples. Alternatively, in the other 9 cases the genotypes of the tumor pooled-gland samples were consistent with those of the corresponding tumor single-gland samples (Figs. 2 and 3 ).

In the samples of intestinal metaplastic glands, although no genetic alterations were found in the pooled-gland samples, multiple alterations were frequently detected in single-gland samples (Fig. 4). A representative example of allelic imbalance at each chromosomal locus in a pooled-gland sample and in corresponding intestinal metaplastic single-gland samples is illustrated in Fig. 5 (case C).

In the samples of nonmetaplastic glands, although no genetic alterations were found in the pooled-gland samples, alterations were observed in corresponding single-gland samples (Fig. 6).
Heterogeneous genotypes in individual glands within the same sample. A total of 18 carcinomas $(18 / 20,90 \%)$ consisted of heterogeneous single genotypes ( $>1$ different genotype) within the same tumor. Single glands within the same sample having the same genotype were interpreted as an occurrence of the same subclone. The number of subclones within the same sample was determined and is shown in Table III. Many subclones were identified within the same sample. No more than 7 different genotypes (subclones) in single-tumor glands from the same sample were observed. In addition, multiple subclones within the same sample of not only intestinal metaplastic single glands but also nonmetaplastic single glands were found. The mean numbers of genetic alterations per tumor, intestinal metaplastic, and nonmetaplastic single-gland samples were $4.15,3.75$ and 4.5 , respectively.

Two samples (10\%) had homogeneous single genotypes within the same tumor (A and N, Figs. 2 and 3). In contrast, MSI did not occur in a homogeneous pattern within the same tumor (G and Q, Figs. 2 and 3). There were no homogeneous patterns observed among either metaplastic or nonmetaplastic single-gland samples (Figs. 4 and 6).

Genotypic pattern of individual glands within the same sample. As shown in our previous study on colorectal carcinoma (3), the genotype of each single-tumor gland was classified as either a major-altered or a minor-altered genotype. The first group, the major-altered genotype, was defined as a single-tumor gland with $>1$ genetic alteration. In contrast, the minor-altered genotype was defined as a single-tumor gland with none or 
$t+$
aldoll

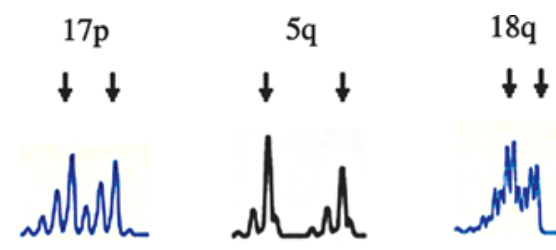

$3 \mathrm{p}$

$4 \mathrm{p}$

$9 \mathrm{p}$

$P$

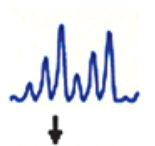<smiles>CCCCCCC(C)C</smiles>

N

1<smiles>C#CCCCCC#C</smiles><smiles>CC1CCCC1C</smiles>

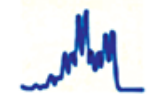<smiles>C#CC</smiles><smiles>C=C1CCCC(C)=C1C</smiles><smiles>CCC(C)C</smiles><smiles>CC(C)/C=C\C(C)C</smiles><smiles>[C+]1CCCC1</smiles>

2

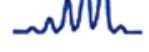<smiles>CCCCC(C)C</smiles><smiles>CC=CC</smiles><smiles>CC(C)C(C)C(C)C</smiles><smiles>C1=CCC1</smiles>

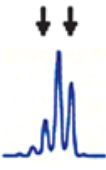

3

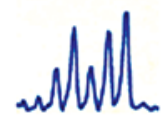<smiles>CCCC1CCCCC1C</smiles><smiles>CCCC</smiles><smiles>CC=CCC</smiles><smiles>CC=CC</smiles>

4

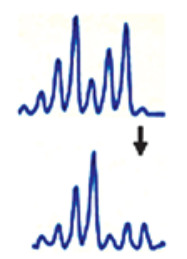<smiles>CCCC1C=C1C</smiles>

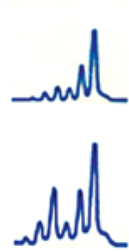<smiles>CCC1C=CCCC1CC</smiles><smiles>CC#CC</smiles>

5<smiles>CC1CC1</smiles><smiles>[AlH2]</smiles><smiles>CC#CCNC(C)C#CC</smiles>

Figure 5. Allelic imbalances on 17p, 5q, 18q, 3p, 4p, and 9p chromosomal loci in an intestinal metaplastic pooled-gland sample and in corresponding intestinal metaplastic single-gland samples (case $\mathrm{C}$ ). Multiple subclones (genotypes) were seen. Note that although no genetic alterations were detected in the pooled-gland sample, they were frequently found in the paired single intestinal metaplastic gland sample. In intestinal metaplastic single-gland samples, arrows indicate a lost allele at each chromosomal locus. $\mathrm{P}$, pooled gland sample; $\mathrm{N}$, normal.

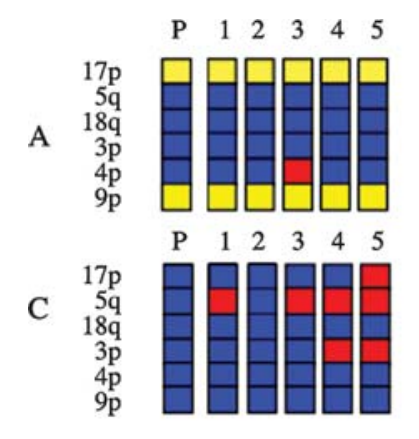

$\begin{array}{llllll}\mathrm{P} & 1 & 2 & 3 & 4 & 5\end{array}$
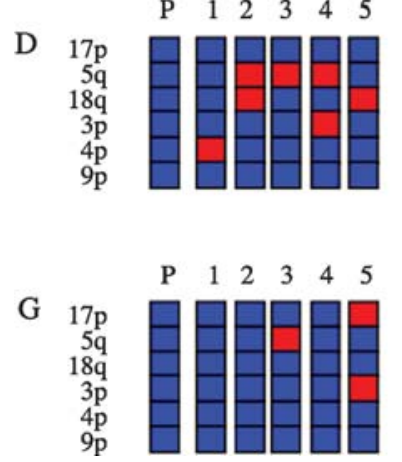

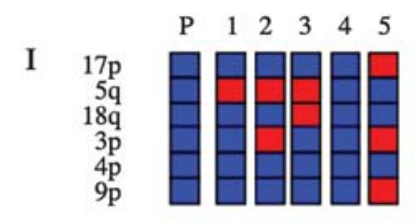

$\begin{array}{llllll}\mathrm{P} & 1 & 2 & 3 & 4 & 5\end{array}$

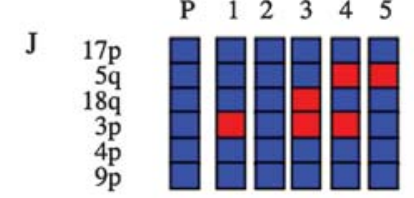

$\begin{array}{llllll}\mathrm{P} & 1 & 2 & 3 & 4 & 5\end{array}$

L 1

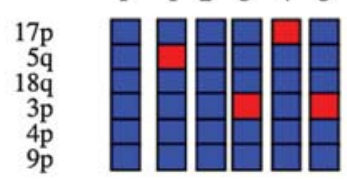

$\begin{array}{llllll}P & 1 & 2 & 3 & 4 & 5\end{array}$

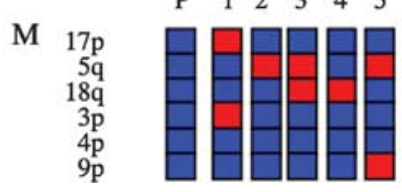

Figure 6. A detailed view of allelic imbalances on 5q, 17p, 18q, 3p, 4p, 9p, and microsatellite instability in nonmetaplastic pooled-gland samples and in corresponding nonmetaplastic single-gland samples (cases: A, C, D, G, I, J, L, M, O, and S). Genetic alterations were seen in the single intestinal metaplastic gland samples. The frequency of allelic imbalances in single nonmetaplastic gland samples is low compared with those of single intestinal metaplastic gland samples. P, pooled gland sample; LOH, loss of heterozygosity; N, negative; NI, not informative; NA, not amplified. 
Table III. The number of subclones within the same sample.

\begin{tabular}{lccc}
\hline \multirow{2}{*}{$\begin{array}{l}\text { Number of } \\
\text { subclones within } \\
\text { the same tumor }\end{array}$} & $\begin{array}{c}\text { Tumor } \\
\text { gland }\end{array}$ & $\begin{array}{c}\text { Intestinal } \\
\text { metaplastic } \\
\text { gland }\end{array}$ & $\begin{array}{c}\text { Nonmetaplastic } \\
\text { gland }\end{array}$ \\
\hline Two & 3 & 2 & 1 \\
Three & 3 & 6 & 1 \\
Four & 3 & 7 & 3 \\
Five & 2 & 5 & 5 \\
Six & 5 & 0 & 0 \\
Seven & 2 & 0 & 0 \\
\hline
\end{tabular}

with 1 genetic alteration. The major-altered genotype contained 2 types of genetic alterations: multiple AI (AI-type) and MSI. Of 20 carcinomas, 12 contained single-tumor glands with the minor-altered genotype within the same tumor. In contrast, all samples of intestinal metaplastic single glands contained the minor-altered genotype with the exception of one case (sample O). However, surprisingly, at least 1 metaplastic single gland with the major-altered genotype metaplastic glands was detected in 18 of 20 samples. Among the nonmetaplastic single-gland samples, there were major-altered genotypes found in 8 of 10 samples.

Classification of genotype alterations in a single gland within individual tumor. Similar to our previous study (3), we categorized the samples we examined (gastric cancers, intestinal metaplastic glands, nonmetaplastic glands, and including homogeneous tumors) into 5 groups according to criteria based on proportion of genotype alteration: I, all major-altered genotypes; II, major-altered > minor-altered genotypes; III, major-altered = minor-altered genotypes; IV minor-altered > major-altered genotypes; V, all minor-altered genotypes. According to these criteria, of single glands from tumors, 8 samples were categorized as type I, 8 as type II, and 3 tumors as type IV. The remaining tumor was classified as type III. No tumor was type V. The 2 tumor samples with MSI were grouped as type II and IV, respectively. In contrast, among samples of intestinal metaplastic single glands, 1 was classified as type I, and 9 and 8 samples were categorized as type II and IV, respectively. Two samples were classified as type V. Among nonmetaplastic gland samples, 2 were classified as type II, 2 as types V, and 6 as type IV.
We looked for any association between the genotypes of pooled-gland samples (major-altered and minor-altered genotypes) and the genotypic patterns (I-V) of tumor, intestinal metaplastic, and nonmetaplastic single-gland samples; results are shown in Table IV. In 3 tumor samples (Q, R, and S), the genotype in pooled-gland samples was not consistent with the genotypic patterns of tumor single-gland samples. In contrast, in 10 intestinal metaplastic samples (B, C, D, E, I, J, K, N, O, and $\mathrm{Q}$ ), the genotype of pooled-gland samples in GIM was different from the genotypic patterns of intestinal metaplastic single-gland samples. In addition, in 7 nonmetaplastic samples (C, D, I, J, M, O, and S), the genotype of pooledgland samples in nonmetaplastic glands was not consistent with the genotype pattern of nonmetaplastic single-gland samples.

\section{Discussion}

In the present study, 12 of 20 tumors showed different predominant genotypes between pooled-gland and single-gland tumor samples. These 12 tumors were primarily classified into 2 groups as an explanation of the discrepancy between pooled-gland and single-tumor gland samples (3). The first explanation is that the AIs in pooled-gland samples at the chromosomal loci we examined may be caused by dilution of minor genotypes within the same tumor (dilution effect). This may represent a mean value of AIs for all collected singlegland tumors. We concluded that the results for 4 tumors (samples: F, I, J, and L) are consistent with this explanation. An alternative explanation may be that the composition of tumor glands in the pooled-gland samples is different from that observed in the single-gland tumor sample (i.e., different composition effect). This suggests that additional subclones, different from those found in the pooled-gland tumor sample, can exist within the same tumor. According to this explanation, it may be likely that a different subclone or different composition of a single-gland sample will not be detected in the pooled-gland samples (B, D, H, O, P, Q, R, and S).

Such a discrepancy between pooled-gland and singlegland samples was prominent in the nonmetaplastic samples. Although no genetic alterations were detected in the pooledgland samples, multiple genetic alterations were found in corresponding single-gland samples. In particular, this finding was frequently present in GIM samples.

In the present study, it appears that isolation of single intestinal metaplastic glands can be used to increase the sensitivity of tests for allelic imbalance. This finding suggests

Table IV. Frequencies of the genotypic patterns of single-gland samples in gastric tumor, gastric intestinal metaplastic glands, and nonmetaplastic glands according to the genotype of pooled-gland samples.

\begin{tabular}{|c|c|c|c|c|c|c|c|c|c|c|c|c|c|c|c|}
\hline \multirow{2}{*}{$\begin{array}{l}\text { Genotype of pooled- } \\
\text { gland samples }\end{array}$} & \multicolumn{5}{|c|}{ Tumor (n-20) } & \multicolumn{5}{|c|}{$\begin{array}{l}\text { Intestinal metaplastic } \\
\text { gland }(n-20)\end{array}$} & \multicolumn{5}{|c|}{$\begin{array}{l}\text { Nonmetaplastic gland } \\
\qquad(\mathrm{n}-10)\end{array}$} \\
\hline & I & II & III & IV & $\mathrm{V}$ & I & II & III & IV & V & I & II & III & IV & V \\
\hline Major & 8 & 6 & 0 & 1 & 0 & 0 & 0 & 0 & 0 & 0 & 0 & 0 & 0 & 0 & 0 \\
\hline Minor & 0 & 2 & 1 & 2 & 0 & 1 & 9 & 0 & 8 & 2 & 0 & 2 & 0 & 6 & 2 \\
\hline
\end{tabular}


that genetic analysis of single intestinal metaplastic glands may be helpful in detecting such genetic alterations.

Marked clinical and biological heterogeneity has been noted among human gastric cancers (1-3). However, possible genetic causes of genetic heterogeneity have not been fully investigated. In this study, we used tumor single-gland samples to look for heterogeneous populations within the same tumor. Our findings that $90 \%$ of tumors demonstrated heterogeneous composition within the same tumor, show that gastric cancers are genetically highly complex. On average, there were 4.2 genetic alterations per tumor. The high number of genetic alterations per tumor indicates that genetic instability may cause intratumoral heterogeneity, as seen in other human tumors $(1,3,27,28)$, and be an underlying mechanism of gastric carcinogenesis. In addition, our findings suggest that a specific subclone cannot be selected in most gastric cancers during tumor progression. This is an important finding toward understanding the effectiveness of chemotherapy or radiotherapy in gastric cancers, because the existence of heterogeneous populations indicates no single target cell can be defined.

The degree of accumulated LOH (allelic imbalance) has been shown to be of prognostic value in various cancer types including gastric cancer, and a high degree of tumor LOH has been shown to be associated with tumor aggressiveness and a worse prognosis (29). In light of these findings, low and high rates of tumor LOH suggest low and high tumor behavioral aggressiveness, respectively. In some of the present cases, although minor-altered genotype (low LOH) was found in the pooled-gland sample, major-altered genotype (high LOH) was detected in the corresponding tumor singlegland sample. Our findings also indicate that 2 of 5 tumors showing a minor-altered genotype in the pooled-gland sample were classified as type II (composed of major-altered genotype) in the tumor single-gland sample. This finding suggests that highly aggressive subclones may exist within a tumor showing a low frequency of $\mathrm{LOH}$ in a pooled-gland sample $(2 / 5,40 \%)$. It is surprising that $40 \%$ of pooled-gland samples showing the minor-altered genotype contained a major-altered genotype in the corresponding tumor singlegland samples. On the other hand, in the present study, most of the carcinomas that were examined contained a minoraltered genotype of single tumor glands within the same tumor. One recent study has shown that high-LOH tumors may have a higher rate of response to chemotherapy $(29,30)$. However, in general, the majority of patients with gastrointestinal cancers are thought to obtain a poor response to chemotherapy without survival benefit $(29,30)$. Although the genetic reason for the discrepancy remains unknown, an explanation may be that the minor-altered genotype gland is a supply source to the major-altered genotype gland. Therefore, a minor-altered genotype gland may co-exist with a majoraltered genotype gland within the same tumor (3).

In the present study, genotypic pattern for a single-gland sample was classified into 5 groups. Although types I and II were the most frequent genotypic patterns $(17 / 20,85 \%)$, type IV, a tumor genotype showing predominantly minor-altered glands, was relatively rare $(3 / 20,15 \%)$. These findings suggest that single tumor glands with multiple genetic alterations may cause subclonal expansions in different areas within the same tumor, leading to occupation of the whole tumor mass in gastric cancers.

The general strategy of identification of individuals at high risk for progression to cancer offers promising possibilities for cancer prevention, and this approach largely depends on early detection. Therefore, it is important to evaluate GIM, which is generally thought to be a precancerous condition in gastric cancer $(12,18)$. Furthermore, a previous study has shown that GIM is closely associated with Helicobacter pylori infection (31). However, little is known about the genetic events responsible for initiation and progression of gastric cancer $(32,33)$. According to an investigation by Ochiai et al, although $p 53$ mutations known to play a key role in human neoplastic progression were identified in GIM, they were detected in only 10 of $756(1.3 \%)$ histological sections (18). This finding indicates that it is difficult to identify such subtle genetic alterations in intestinal metaplastic glands. Genetic analysis of a single gland may enable us to identify subtle genetic alterations in GIM. Therefore, we used isolated single glands in the current study to address the issue of whether molecular alterations occur in GIM. In this study, although no genetic alterations were detected in pooled samples of intestinal metaplastic glands, alterations were frequently found in the corresponding single intestinal metaplastic gland samples. This suggests that intestinal metaplastic glands have a markedly heterogeneous composition. This study is the first to identify that expansive microsatellite alterations are seen in samples of intestinal metaplastic glands. These data indicate that irreversible genetic changes have already occurred in morphologically non-neoplastic gastric mucosa with intestinal metaplasia, and they support the hypothesis that GIM may be a precursor lesion of gastric cancer.

The present study has demonstrated that multiple genetic alterations are frequently found in nonmetaplastic glands. This is a surprising finding and the first study to identify genetic alterations in histologically normal gastric glands. This finding suggests that accumulation of genetic alterations occurs not only in metaplastic glands but also in nonmetaplastic glands, and that genetic alterations in gastric epithelial cells during chronic gastritis may contribute to an increased risk of gastric cancer (34).

In conclusion, single tumor glands can be useful for investigating genetic alterations in gastric cancers and gastric intestinal metaplasia. The present data indicate that most carcinomas and GIM are genetically heterogeneous. Recently, public policy strategies have been suggested for identification of patients at risk for $H$. pylori-related gastric malignancy $(35,36)$. The thrust of this policy is that eradication of $H$. pylori infection earlier rather than later in life is anticipated to be more beneficial, because gastric intestinal metaplasia is expected to occur later in life. Our finding that multiple genetic alterations are found in single intestinal metaplastic glands may support this opinion.

\section{Acknowledgements}

We gratefully acknowledge the technical assistance of Miss E. Sugawara and Mr. T. Kasai. We also thank members of the Division of Pathology, Central Clinical Laboratory, Iwate Medical University, for their support. 


\section{References}

1. Nowel PC: The clonal evolution of tumor cell populations Science 194: 23-28, 1976.

2. Ishii M, Sugai T, Habano W and Nakamura S: Analysis of Ki-ras gene mutations within the same tumor using a single tumor crypt in colorectal carcinomas. J Gastroenterol 39: 544-549, 2004

3. Sugai T, Habano W, Jiao Y-F, et al: Analysis of allelic imbalances at multiple cancer-related chromosomal loci and microsatellite instability within the same tumor using a single tumor gland from colorectal carcinomas. Int J Cancer 114: 337-345, 2005.

4. Fearon ER and Vogelstein B: A genetic model for colorectal tumorigenesis. Cell 61: 759-767, 1990.

5. Chung YJ, Kim KM, Choi JR, Choi SW and Rhyu MG: Relationship between intratumor histological heterogeneity and genetic abnormalities in gastric carcinoma with microsatellite instability. Int J Cancer 82: 782-788, 1999.

6. Iwamatsu H, Nishikura K, Watanabe H, et al: Heterogeneity of p53 mutational status in the superficial spreading type of early gastric carcinoma. Gastric Cancer 4: 20-26, 2001.

7. Sugai T, Uesugi N, Habano W, et al: DNA mapping of gastric cancers using flow cytometric analysis. Cytometry 42: 270-276, 2000.

8. Nakayama S, Nakayama K, Takebayashi Y, et al: Allelotypes as potential prognostic markers in ovarian carcinoma treated with cisplatin-based chemotherapy. Int J Mol Med 11: 621-625, 2003.

9. Barratt PL, Seymour MT, Stenning SP, et al: UKCCCR AXIS trial collaborators. Adjuvant X-ray and Fluorouracil Infusion Study. UKCCCR AXIS trial collaborators. DNA markers predicting benefit from adjuvant fluorouracil in patients with colon cancer: a molecular study. Lancet 360: 1381-1391, 2002.

10. Day DW, Jass JR, Price AB, Shepherd NA, Sloan JM, Talbot IC, Warren BF and Williams GT (eds): Morson and Dawson's Gastrointestinal Pathology. 4th edition, Blackwell Science, 114-115, 2003.

11. Mutoh H, Sakurai S, Satoh K, et al: Development of gastric carcinoma from intestinal metaplasia in $\mathrm{Cdx} 2$-transgenic mice. Cancer Res 64: 7740-7747, 2004.

12. Morgan C, Jenkins GJ, Ashton T, et al: Detection of p53 mutations in precancerous gastric tissue. Br J Cancer 89: 1314-1319, 2003.

13. Tatematsu M, Tsukamoto $\mathrm{T}$ and Mizoshita T: Role of Helicobacter pylori in gastric carcinogenesis: The origin of gastric cancers and heterotopic proliferative glands in Mongolian Gerbils. Helicobacter 10: 97-106, 20056.

14. Baracchini P, Fulcheri E and Lapertosa G: Patterns of intestinal metaplasia in gastric biopsies. A comparison of different histochemical classifications. Histochem J 23: 1-9, 1991.

15. Matsukura N, Suzuki K, Kawachi T, et al: Distribution of marker enzymes and mucin in intestinal metaplasia in human stomach and relation to complete and incomplete types of intestinal metaplasia to minute gastric carcinomas. J Natl Cancer Inst 65 : 231-240, 1980 .

16. Mirza ZK, Das KK, Slate J, et al: Gastric intestinal metaplasia as detected by a monoclonal antibody is highly associated with gastric adenocarcinoma. Gut 52: 807-812, 2003.

17. Blok P, Craanen ME, Offerhaus GJ and Tytgat GN: Gastric carcinoma: clinical, pathogenic, and molecular aspects. QJM 90: 735-749, 1997

18. Ochiai A, Yamauchi Y and Hirohashi S: p53 mutations in the non-neoplastic mucosa of the human stomach showing intestinal metaplasia. Int J Cancer 69: 28-33, 1996.

19. Sugai T, Habano W, Nakamura S, et al: Genetic alterations in DNA diploid, aneuploid and multiploid colorectal carcinomas identified by the crypt isolation technique. Int J Cancer 88 : 614-619, 2000
20. Arai T and Kino I: Morphometrical and cell kinetic studies of normal human colorectal mucosa: Comparison between the proximal and the distal large intestine. Acta Pathol Jpn 39: 725-730, 1989.

21. Japanese Research Society for Gastric Cancer: The General Rules for the Gastric Cancer Study. 12th edition, Kanehara-Shuppan, Tokyo, pp64-89, 1993.

22. Nakamura S, Goto J, Kitayama M and Kino I: Application of the crypt-isolation technique to flow-cytometric analysis of DNA content in colorectal neoplasms. Gastroenterology 106: 100-107, 1994.

23. Sugai T, Habano W, Uesugi N, et al: Three independent genetic profiles based on mucin expression in early differentiated-type gastric cancers - a new concept of genetic carcinogenesis of early differentiated-type adenocarcinomas. Mod Pathol 17: 1223-1234, 2004.

24. Jiao Y-F, Sugai T, Habano W, Suzuki M, Takagane A and Nakamura S: Analysis of microsatellite alterations in gastric carcinoma by applying the crypt isolation technique. J Pathol 204: 200-207, 2004

25. Habano W, Sugai T, Nakamura S and Yoshida T: A novel method for gene analysis of colorectal carcinomas using a crypt isolation technique. Lab Invest 74: 933-940, 1996.

26. Sugai T, Takahashi H, Habano W, et al: Analysis of genetic alterations, classified according to their DNA ploidy pattern, in the progression of colorectal adenomas and early colorectal carcinomas. J Pathol 200: 168-176, 2003.

27. Faquin WC, Fitzgerald JT, Boynton KA and Mutter GL: Intratumoral genetic heterogeneity and progression of endometrioid type endometrial adenocarcinomas. Gynecol Oncol 78: 152-157, 2000.

28. Matsumoto T, Fujii H, Arakawa A, et al: Loss of heterozygosity analysis shows monoclonal evolution with frequent genetic progression and divergence in esophageal carcinosarcoma. Hum Pathol 35: 322-327, 2004.

29. Grundei T, Mueller J, Scholz M, et al: Loss of heterozygosity and microsatellite instability as predictive markers for neoadjuvant treatment in gastric carcinoma. Clin Cancer Res 6: 4782-4788, 2000 .

30. Ott K, Vogelsang H, Ott K, et al: Chromosomal instability rather than p53 mutation is associated with response to neoadjuvant cisplatin-based chemotherapy in gastric carcinoma. Clin Cancer Res 9: 2307-2315, 2003.

31. Hirayama F, Takagi S, Yokoyama Y, Yamamoto K, Iwao E and Haga K: Long-term effects of Helicobacter pylori eradication in Mongolian gerbils. J Gastroenterol 37: 779-784, 2002.

32. Boussioutas A, Li H, Liu J, et al: Distinctive patterns of gene expression in premalignant gastric mucosa and gastric cancer. Cancer Res 63: 2569-2577, 2003.

33. Sugai T, Habano W, Nakamura S, et al: Correlation of histological morphology and tumor stage with molecular genetic analysis using microdissection in gastric carcinomas. Diagn Mol Pathol 7: 235-240, 1998.

34. Yao Y, Tao H, Park DI, Sepulveda JL and Sepulveda AR: Demonstration and characterization of mutations induced by Helicobacter pylori organisms in gastric epithelial cells. Helicobacter 11: 272-286, 2006.

35. Hunt RH: Will eradication of Helicobacter pylori infection influence the risk of gastric cancer? Am J Med 117 (Suppl 5A): S86-S91, 2004.

36. Yoo EJ, Park SY, Cho NY, Kim N, Lee HS and Kang GH: Helicobacter pylori-infection-associated $\mathrm{CpG}$ island hypermethylation in the stomach and its possible association with polycomb repressive marks. Virchows Arch 452: 515-524, 2008 . 